\title{
Análise clínica e radiográfica de uma pasta endodôntica à base de hidróxido de cálcio
}

\section{e Aloe vera: ensaio clínico randomizado}

\author{
Clinical and radiographic analysis of an endodontic paste based on calcium hydroxide and Aloe \\ vera: randomized clinical trial \\ Análisis clínico y radiográfico de una pasta endodóntica a base de hidróxido de calcio y Aloe vera:
} ensayo clínico aleatorizado

\author{
Larissa Cordeiro Cavalcante \\ ORCID: https://orcid.org/0000-0002-4838-7473 \\ Universidade Federal do Piauí, Brasil \\ E-mail: larissacordeirocavalcante@gmail.com \\ Isadora Mello Vilarinho Soares \\ ORCID: https://orcid.org/0000-0002-0902-707X \\ Universidade Federal do Piauí, Brasil \\ E-mail: isadoramvsoares@hotmail.com \\ Carmen Milena Rodrigues Siqueira Carvalho \\ ORCID: https://orcid.org/0000-0001-9191-1499 \\ Universidade Federal do Piauí, Brasil \\ E-mail: carmenmilena@ufpi.edu.br
}

\begin{abstract}
Resumo
Esse ensaio clínico randomizado avaliou uma pasta medicamentosa intracanal à base de $\mathrm{Ca}(\mathrm{OH})_{2}$ e Aloe vera em dentes necrosados com lesão periapical. Sessenta e quatro dentes, de 60 pacientes, foram divididos de acordo com a medicação intracanal utilizada: Controle $\left(\mathrm{Ca}(\mathrm{OH})_{2}\right.$ e soro fisiológico), e experimental $\left(\mathrm{Ca}(\mathrm{OH})_{2}\right.$ e Aloe vera). Foi realizado instrumentação endodôntica e inserção da medicação intracanal de acordo com o grupo. Após 15 dias, os dentes foram obturados e restaurados. A sintomatologia pós-operatória foi avaliada seguindo uma escala de dor. A avaliação clínica e radiográfica foi realizada aos três, seis e nove meses pós-tratamento. Os tratamentos foram classificados em sucesso (os que apresentaram cicatrização parcial ou total) e insucesso. Para análise estatística de dor pós-operatória entre grupos foi utilizado o teste Mann-Whitney. A análise intragrupo utilizou o teste de Friedmann. Para avaliação do sucesso do tratamento, utilizou-se o teste qui-quadrado. Considerou-se significativo $\mathrm{p}<0,05$. Quarenta e oito pacientes, sendo um dente em cada, concluíram o estudo. Os valores da dor pós-operatória nos tempos 04 e 24 horas para significativos para o grupo Aloe vera $(\mathrm{p}<0,001)$, sem diferença estatística entre os grupos $(\mathrm{p}=0,307)$ no tempo de 48 horas. Após os nove meses, as taxas de sucesso foram de $95,8 \%$ para o grupo experimental e $83,3 \%$ para o controle, sem diferença significativa $(\mathrm{p}=0,348)$. A pasta de Aloe vera e $\mathrm{Ca}(\mathrm{OH})_{2}$ proporcionou remissão da dor pós-operatória, sem alterações clínicas e radiográficas significativas. Acredita-se que o Aloe vera pode ser um veículo alternativo para o hidróxido de cálcio como medicação intracanal.
\end{abstract}

Palavras-chave: Hidróxido de cálcio; Aloe; Terapia de canal radicular.

\begin{abstract}
This randomized clinical trial evaluated an intracanal medicinal paste based on $\mathrm{Ca}(\mathrm{OH}) 2$ and Aloe vera in necrotic teeth with periapical lesions. Sixty-four teeth, from 60 patients, were divided according to the intracanal medication used: Control $(\mathrm{Ca}(\mathrm{OH}) 2$ and saline solution), and experimental $(\mathrm{Ca}(\mathrm{OH}) 2$ and Aloe vera). Endodontic instrumentation and insertion of intracanal medication were performed according to the group. After 15 days, the teeth were filled and restored. Postoperative symptoms were assessed following a pain scale. Clinical and radiographic evaluation was performed at three, six and nine months after treatment. Treatments were classified into success (those with partial or complete healing) and failure. For statistical analysis of postoperative pain between groups, the Mann-Whitney test was used. The intragroup analysis used the Friedmann test. To assess treatment success, the chi-square test was used. It was considered significant $\mathrm{p}<0.05$. Forty-eight patients, one tooth in each, completed the study. Postoperative pain values at 04 and 24 hours were significant for the Aloe vera group $(\mathrm{p}<0.001)$, with no statistical difference between groups $(\mathrm{p}=0.307)$ at 48 hours. After nine months, success rates were $95.8 \%$ for the experimental group and $83.3 \%$ for the control, with no significant difference $(\mathrm{p}=0.348)$. Aloe vera and $\mathrm{Ca}(\mathrm{OH}) 2$ paste provided remission of postoperative pain, without significant clinical and radiographic changes. Aloe vera is believed to be an alternative vehicle for calcium hydroxide as an intracanal medication.
\end{abstract}

Keywords: Calcium hydroxide; Aloe; Root channel therapy. 


\section{Resumen}

Este ensayo clínico aleatorizado evaluó una pasta medicinal intracanal a base de $\mathrm{Ca}(\mathrm{OH}) 2$ y Aloe vera en dientes necróticos con lesiones periapicales. Se dividieron 64 dientes, de 60 pacientes, según la medicación intracanal utilizada: Control $(\mathrm{Ca}(\mathrm{OH}) 2$ y solución salina) y experimental $(\mathrm{Ca}(\mathrm{OH}) 2$ y Aloe vera). La instrumentación endodóntica y la inserción de medicación intracanal se realizaron según el grupo. Después de 15 días, se obturaron y restauraron los dientes. Los síntomas postoperatorios se evaluaron siguiendo una escala de dolor. La evaluación clínica y radiográfica se realizó a los tres, seis y nueve meses después del tratamiento. Los tratamientos se clasificaron en éxito (aquellos con curación parcial o completa) y fracaso. Para el análisis estadístico del dolor postoperatorio entre grupos se utilizó la prueba de Mann-Whitney. El análisis intragrupo utilizó la prueba de Friedmann. Para evaluar el éxito del tratamiento, se utilizó la prueba de chi-cuadrado. Se consideró significativo p <0,05. Cuarenta y ocho pacientes, un diente en cada uno, completaron el estudio. Los valores de dolor posoperatorio a las 04 y 24 horas fueron significativos para el grupo de Aloe vera $(\mathrm{p}<0,001)$, sin diferencia estadística entre los grupos $(\mathrm{p}=0,307)$ a las 48 horas. Después de nueve meses, las tasas de éxito fueron del 95,8\% para el grupo experimental y del 83,3\% para el control, sin diferencias significativas $(\mathrm{p}=0,348)$. La pasta de aloe vera y $\mathrm{Ca}(\mathrm{OH}) 2$ proporcionaron la remisión del dolor postoperatorio, sin cambios clínicos y radiográficos significativos. Se cree que el aloe vera es un vehículo alternativo para el hidróxido de calcio como medicamento intracanal.

Palabras clave: Hidróxido de calcio; Áloe; Terapia de conductos radiculares.

\section{Introdução}

A lesão periapical é uma resposta imunoinflamatória localizada decorrente dos micro-organismos advindos do sistema de canais radiculares (SCR) contaminados em direção aos tecidos apicais e periapicais, com consequente reabsorção óssea e dentária (Liapatas; Nakou; Rontogianni, 2003; Nair, 2006). Visando o reparo periapical, se faz necessária a eliminação ou redução dos micro-organismos e seus subprodutos do SCR e total obturação dos condutos. Contudo, este objetivo é difícil de ser alcançado pelo preparo biomecânico devido à complexidade das variações anatômicas dos dentes, como istmos, ramificações, reentrâncias, achatamentos, deltas apicais e canais acessórios (Paqué; Ganahl; Peters, 2009; Rezende et al., 2016).

Devido à inacessibilidade dos instrumentos nas áreas de complexidade anatômica, soluções irrigadoras, através de suas ações química, física e mecânica, são utilizadas como coadjuvantes à limpeza e desinfecção do SCR (Van der Sluis; Versluis; Wesselink, 2007), atingindo, assim, as áreas fora do alcance dos instrumentos endodônticos. Para auxiliar no controle e erradicação de infecções, medicações intracanais são utilizadas no intuito de reduzir as bactérias remanescentes, seus metabólitos, e acelerar o processo de cicatrização (Rezende et al., 2016). O hidróxido de cálcio $\left(\mathrm{Ca}(\mathrm{OH})_{2}\right)$ tem sido largamente utilizado para este propósito devido sua ação antimicrobiana resultante de sua alta alcalinidade. $\mathrm{Além} \mathrm{disso,} \mathrm{o} \mathrm{Ca}(\mathrm{OH})_{2}$ apresenta propriedades anti-inflamatórias, capacidade de indução de mineralização e reparo apical, e biocompatibilidade (Mohammadi; Dummer, 2011; Batista; Olian; Mori, 2014; Abbaszadegan et al., 2016; Sholehvar et al., 2016).

Diferentes substâncias podem ser empregadas como veículo para o $\mathrm{Ca}(\mathrm{OH})_{2}$, as quais podem melhorar ou modificar suas propriedades, influenciando na sua difusão ou dissociação iônica (Batista; Olian; Mori, 2014), como por exemplo, soro fisiológico, glicerina, anestésico e clorexidina (Vianna et al., 2009). Novos veículos têm sido estudados no intuito de promoverem melhores propriedades à medicação intracanal, dos quais, destacam-se, os fitoterápicos (Batista; Olian; Mori, 2014; Abbaszadegan et al., 2016).

Recentemente, o uso de fitoterápicos em odontologia tem crescido exponencialmente. O Aloe vera (Aloe barbadensis Miller), membro da família das Liliaceae, tem sido avaliado nos mais diversos campos da odontologia, dos quais destacam-se sua atuação na cicatrização de lesões orais (Ali; Wahbi, 2017). Popularmente conhecida como babosa, a espécie Aloe vera contém diversas substâncias biologicamente ativas, capazes de proporciornar importantes propriedades anti-inflamatórias, antibacterianas, hipoglicêmicas, imunomoduladoras, cicatrizantes e regenerativas (Abbaszadegan et al., 2016; Jittapiromsak et al., 2010; Fani; Kohanteb, 2012; Fé et al., 2014). 
Na Endodontia, Batista et al. (2014), por meio de um estudo in vitro, relatou o uso do Aloe vera como veículo para o $\mathrm{Ca}(\mathrm{OH})_{2}$ como medicação intracanal e evidenciou que o uso desse fitoterápico possibilita a dissociação iônica do $\mathrm{Ca}(\mathrm{OH})_{2}$ através dos túbulos dentinários. Até o momento, não foram relatados na literatura estudos clínicos sobre o tema.

Dessa forma, o objetivo deste ensaio clínico randomizado foi avaliar a efetividade de uma pasta medicamentosa intracanal à base de $\mathrm{Ca}(\mathrm{OH})_{2}$ e Aloe vera em dentes necrosados com lesão periapical visível radiograficamente. A hipótese testada é que o Aloe vera reduz a sintomatologia pós-operatória e acelera a cicatrização radiográfica.

\section{Métodos}

\section{Aspectos éticos}

O Projeto foi submetido à apreciação pelo Comitê de Ética em Pesquisa (CEP) da Universidade Federal do Piauí (UFPI), com parecer favorável $n^{\circ}$ (Parecer: 1.835.996). Os participantes da pesquisa, assinaram o Termo de Consentimento Livre e Esclarecido, de acordo com a Resolução do Conselho Nacional de Saúde 466/12, que regulamenta as diretrizes de pesquisa envolvendo seres humanos.

Foi solicitada autorização prévia da Diretoria do Centro Integrado de Saúde Lineu Araújo, onde funciona o Centro de Especialidades Odontológicas I - Teresina- Piauí, para a realização da pesquisa.

O estudo foi submetido ao Registro Brasileiro de Ensaios Clínicos (ReBEC), com o protocolo (REQ:4405).

\section{Desenho do estudo}

Trata-se de um ensaio clínico randomizado, controlado, duplo-cego e paralelo.

\section{Cálculo amostral}

O tamanho da amostra foi calculado por amostragem complexa, no programa Statistical Package for the Social Sciences 20.0 (SPSS) (SPSS Inc., Chicago,IL, USA), versão para Windows, a partir de estudo anterior (Singh et al., 2013), com metodologia semelhante a um dos aspectos avaliados. Foram levados em consideração os parâmetros (média e desviopadrão) obtidos por Singh et al. (2013) e o efeito do desenho amostral desse estudo.

Segundo Silva (2011), para calcular o tamanho total da amostra, deve-se escolher o grupo do estudo que apresenta a maior variância e, nesse, deve ser considerado a maior amostra obtida. Nesse estudo, foi preconizada a amostra de 48 horas. Com o intervalo de $95 \%$, associado a um erro de 5\%, obteve-se o tamanho da amostra entre 44-64, sendo esses os valores mínimo e máximo para a amostra, respectivamente. O valor inicial da amostra foi, portanto, de 64 dentes, por ser o valor máximo da amostra e par.

\section{População e Amostra}

A população do estudo foi composta de pacientes atendidos no Centro Integrado de Saúde Lineu Araújo, referência em atendimento de especialidades odontológicas no âmbito da saúde pública da cidade de Teresina, Piauí, Brasil. Os pacientes atendidos no centro de referência, com dentes assintomáticos, polpas necrosadas e presença de lesão periapical verificada radiograficamente, foram consecutivamente incluídos no estudo, de acordo com os critérios de inclusão e exclusão, até o fechamento do total da amostra $(n=64)$. Os participantes foram distribuídos em dois grupos de acordo com a medicação intracanal utilizada durante o tratamento endodôntico: controle, no qual foi utilizado $\mathrm{Ca}(\mathrm{OH})_{2}$ e soro fisiológico e experimental, $\mathrm{Ca}(\mathrm{OH})_{2}$ e Aloe vera.

Os critérios de inclusão no estudo eram pacientes adultos, com faixa etária entre 18 e 60 anos, sistematicamente saudáveis, que apresentavam necessidade de tratamento endodôntico em dente com canal único e lesão periapical. Foram 
excluídos pacientes com histórico de alergia aos materiais utilizados na pesquisa, comprometimento de saúde, mulheres grávidas ou lactantes, dentes com histórico de tratamento endodôntico anterior, ou presença de calcificação pulpar, reabsorção interna ou externa.

Para selecionar de forma aleatória o grupo em que cada paciente iria participar, foi utilizado um envelope contendo 64 papéis, sendo 32 identificados com o nome Controle e 32 com o nome Experimental. Após o aceite do paciente em participar do estudo, o mesmo sorteava um papel do envelope referente a que grupo ia compor. Após o tratamento, os participantes foram acompanhados para avaliação por nove meses. Durante a proservação, foram perdidos 14 pacientes por desistência ou gravidez. No final da pesquisa, dois participantes foram excluídos devido suas radiografias não terem alcançado consenso entre os examinadores. Desse modo, 48 foram submetidos a análise, sendo 24 pertencentes ao grupo controle e 24 ao grupo experimental. O fluxograma do estudo é apresentado na Figura 1.

Figura 1. Fluxograma de alocação dos participantes segundo CONSORT 2010.

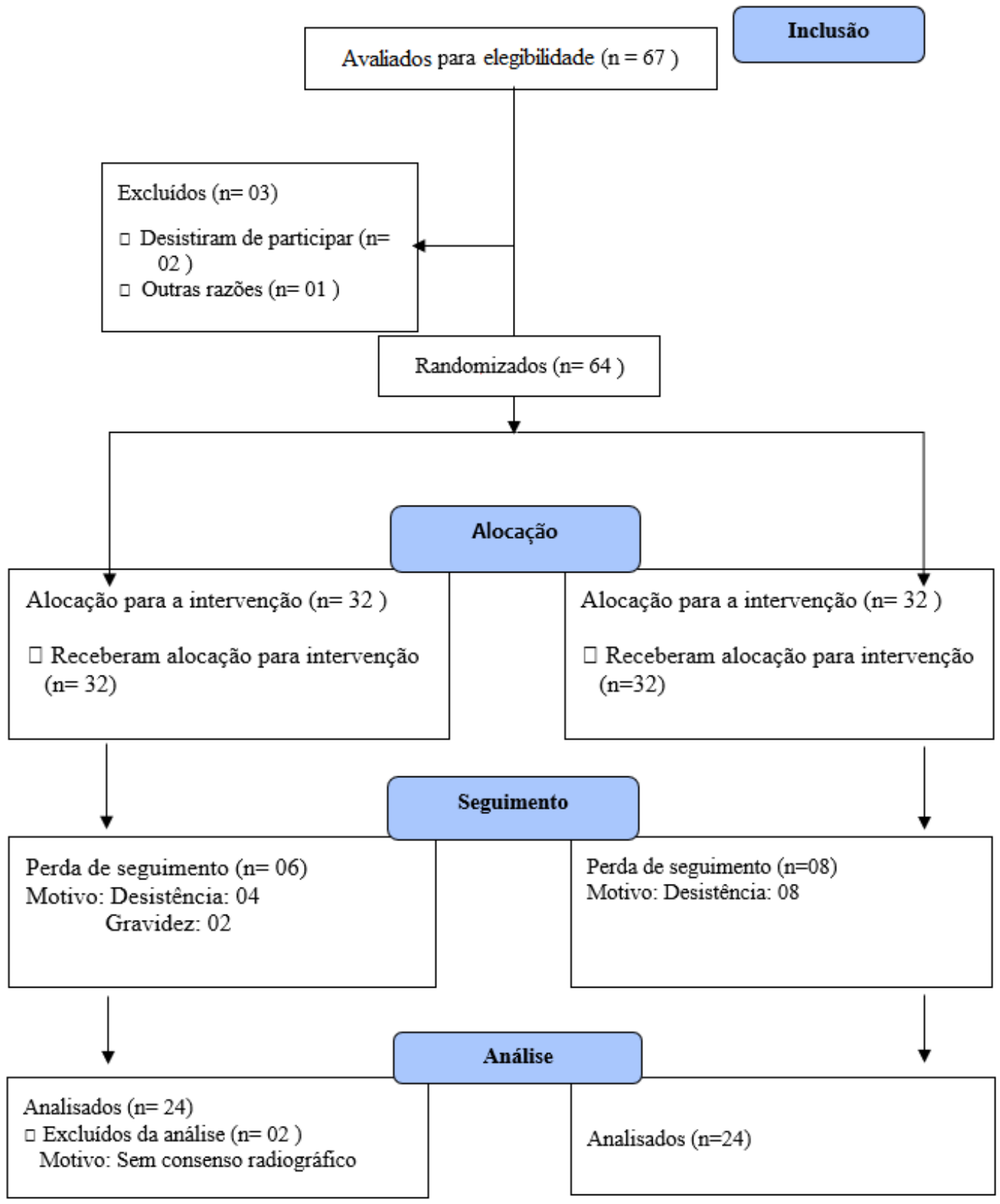

Fonte: Autores. 


\section{Obtenção e Preparo do Extrato de Aloe Vera.}

O extrato de Aloe vera foi obtido da planta Aloe barbadensis Miller, popularmente conhecida como babosa. A planta foi cultivada no Núcleo de Pesquisas em Plantas Medicinais, Centro de Ciências Agrárias, da Universidade Federal do Piauí. Afim de manter a homogeneidade das amostras utilizadas, todas as folhas foram obtidas do mesmo cultivo e sob as mesmas condições de umidade e temperatura.

Para a obtenção do extrato, as folhas foram cortadas a $5 \mathrm{~cm}$ do talo, lavadas em água corrente e colocadas em um recipiente com água destilada por 24 horas com a finalidade de evitar a contaminação do gel com a seiva no momento da extração. Após as 24 horas, as folhas foram novamente lavadas em água corrente, e desinfetadas com etanol a 70\%. Sob condições esteréis, o gel de Aloe vera foi removido por suave raspagem da folha com auxílio de uma espátula $n^{\circ} 24$ (Duflex, Juiz de Fora, Minas Gerais, Brasil), separando o gel interno da casca de folha exterior por meio de uma faca. Em seguida, o gel foi homogeneizado e filtrado em Filtro Disco PureFlo de $25 \mathrm{~mm}$ e micragem igual a 0,45 $\mu \mathrm{m}$ (ZenPure Corporation, Manassas, Virginia, Estados Unidos) e armazenado em tubo falcon sem exposição à luz (Sholehvar et al., 2016).

As coletas da planta e filtração do extrato foram executadas em dias específicos, objetivando manter a padronização do estudo, com amostras coletadas em até vinte e quatro horas. Para transporte do material, os extratos de Aloe vera foram armazenados em tubos falcon cobertos e isentos de calor, para evitar oxidação (Kalra et al., 2017).

\section{Tratamento endodôntico}

A fim de manter a padronização dos procedimentos, o tratamento endodôntico foi realizado em todos os dentes pelo mesmo operador, o qual é Doutor em Endodontia, com experiência na área clínica. O operador não era informado sobre qual medicamento cada paciente estava recebendo.

Para a abertura coronária, foram utilizadas pontas esféricas diamantadas de haste longa para alta rotação numeração 1012HL, 1014HL (KG Sorensen, Cotia, São Paulo, Brasil). Em seguida, irrigou-se abundantemente a câmara pulpar e o orifício de entrada do canal, com solução hipoclorito de sódio 2,5\% (Biodinâmica Química e Farmacêutica, Ibiporã, Paraná, Brasil) por meio de seringa e ponta NaviTip 30G - 25mm (Ultradent Products Inc., South Jordan, UT, EUA), e a partir desta etapa, a irrigação, aspiração e inundação foram constantes, até o término da etapa de instrumentação. A exploração do canal foi então realizada com auxílio de lima \#10, tipo K, de aço inox (Dentsply Maillefer, Ballaigues, Jura-Nord Vaudois, Suíça). Em seguida, a entrada dos canais foi alargada utilizando-se brocas do tipo Gates Glidden (Dentsply Maillefer, Ballaigues, JuraNord Vaudois, Suíça). Posteriormente, realizou-se penetração desinfetante com limas de maior calibre, sendo estas sucessivamente trocadas por menos calibrosas, imprimindo-lhes movimentos oscilolatero-rotatórios no sentido horário até o comprimento de trabalho provisório (comprimento do dente na radiografia de diagnóstico menos $3 \mathrm{~mm}$ ).

Após confirmação do comprimento real do dente por meio de localizador foraminal e radiografia, foi executado a instrumentação com sistema Easy (Easy, Jardinópolis, Belo Horizonte, Minas Gerasi). Em seguida, realizou-se o desbridamento foraminal com lima de menor calibre, irrigação final e a secagem dos canais com cones de papel absorvente correspondentes ao diâmetro do preparo (EndoPoints Industrial da Amazônia LTDA, Manacapuru, Amazonas, Brasil). A medicação intracanal foi preparada no momento do uso e inserida com instrumento manual \#30 ((Dentsply Maillefer, Ballaigues, Jura-Nord Vaudois, Suíça), em movimentos horário e anti-horário, de forma que dois terços das raízes fossem preenchidos com o material. A abertura foi selada, provisoriamente, com cimento de ionômero de vidro (Vitro Fil, DFL Indústria e Comércio S.A., Taquara, Rio de Janeiro, Brasil). 


\section{Grupo Experimental}

A pasta experimental foi preparada na proporção de 1g do pó de $\mathrm{Ca}(\mathrm{OH})_{2}$ (Biodinâmica Química e Farmacêutica, Ibiporã, Paraná, Brasil) para $1 \mathrm{~mL}$ do extrato da Aloe vera, utilizando-se placa de vidro esterilizada e espátula $\mathrm{n}^{\circ} 24$ (Batista; Olian; Mori, 2014).

\section{Grupo Controle}

Seguindo as proporções estabelecidas no mesmo estudo, a pasta controle foi preparada utilizando $1 \mathrm{~g}$ do pó de $\mathrm{Ca}(\mathrm{OH})_{2}$ e 1,5 mL de soro fisiológico estéril (Eurofarma, Itapevi, São Paulo, Brasil), utilizando placa de vidro e espátula $\mathrm{n}^{\circ} 24$ esterilizadas.

Após 15 dias, em todos os casos, os pacientes retornaram sem sintomatologia dolorosa, e os dentes foram obturados pela técnica de condensação lateral utilizando cimento AH-Plus® (Dentsply Maillefer, Ballaigues, Jura-Nord Vaudois, Suíça) e cones de guta-percha (Dentsply Maillefer, Ballaigues, Jura-Nord Vaudois, Suíça). Em seguida, todos os dentes foram restaurados com resina composta (FGM, Joinville, Brasil).

\section{Análise de Sintomatologia pós-operatória}

Cada participante da pesquisa foi convidado a avaliar, conforme a escala analógica de dor (Tabela 3), nos períodos de 4, 24 e 48 horas após conclusão o tratamento endodôntico (Singh et al., 2013)

\section{Análise Clínica}

Durante os retornos trimestrais para avaliação radiográfica, foram avaliados os seguintes critérios clínicos (Zhou et al., 2017):

- Edema;

- $\quad$ Dor à percussão e palpação do dente;

- Tumefação á palpação dos tecidos moles adjacentes;

- $\quad$ Presença de uma fístula associada

- Inchaço na região próxima ao dente afetado.

Os dentes que apresentaram insucesso clínico ou necessidade de retratamento, pelos motivos acima relatados, foram considerados como dentes que não obtiveram cicatrização de lesão periapical.

\section{Exame Radiográfico}

Os elementos dentários pesquisados foram radiografados pela técnica da bissetriz em cinco momentos: antes do início de tratamento endodôntico, ao final, e nos períodos de 3, 6 e 9 meses após o término do tratamento. Para isso, foi utilizado um aparelho de raio-x (Gnatus, Ribeirão Preto, São Paulo, Brasil), posicionador de filmes cone indicador autoclavável (Maquira, Maringá, Paraná, Brasil) e filme radiográfico dental (Kodak, Rochester, Nova Iorque, Estados Unidos). Após posicionado em cadeira odontológica, o paciente foi protegido com avental plumbífero, confeccionado por borracha plumbífera com equivalência $0,25 \mathrm{pb}$. O filme radiográfico odontológico foi levado em posicionador à boca do paciente. Assim, a tomada radiográfica foi realizada com tempo de exposição de 0,7 segundos. As películas foram revelados de forma manual, através da técnica inspecional, utilizando revelador e fixador Kodak (Kodak, Rochester, Nova Iorque, Estados Unidos) e deixadas em ambiente natural para secar.

Os tempos de processamento foram padronizados de acordo com a temperatura ambiente de aproximadamente ou superior a $30^{\circ} \mathrm{C}$ : um minuto no revelador, 20 segundos para enxágue, três minutos no fixador e cinco minutos para lavagem 
final. Todos os exames radiográficos foram realizados e processados pelo mesmo operador, a fim de manter a padronização das imagens.

\section{Análise Radiográfica}

Todos os filmes radiográficos obtidos nos cinco momentos avaliados pré e pós-operatório da pesquisa foram codificados e organizados em envelopes, em ordem aleatória. As radiografias foram avaliadas por dois avaliadores endodontistas independentes, que não sabiam a quais grupos cada exame pertencia.

De acordo com a metodologia proposta nos estudos de Molander et al (2007) e Zhou et al (2017), os avaliadores foram instruídos a avaliar o tamanho da radioluscência periapical medindo, com uma régua (para o milímetro mais próximo), a sua maior largura horizontal e vertical; sem a realização de uma calibração inicial, já que os dois endodontistas avaliaram durante a pesquisa todos os exames. Em casos de desacordo, o consenso foi alcançado por meio de uma discussão conjunta. Os casos em que não houve consenso (dois casos), foram excluídos. Para manter a fidelidade do estudo, ambos usaram o mesmo instrumento de medida.

\section{Classificação dos resultados}

O resultado das avaliações clínicas e radiográficas foi classificado através de uma adaptação dos critérios utilizados por Zhou et al. (2017) e Molander et al (2007). A classificação da cicatrização segue abaixo:

- Cicatrização completa: completa restituição do contorno periodontal, com restabelecimento da lâmina dura.

- Cicatrização incompleta: somatório da redução da lesão periapical horizontal e vertical maior ou igual a 2 mm.

- Sem cicatrização: presença de sintomas clínicos e/ou aumento ou manutenção do tamanho da lesão periapical.

Os dados obtidos foram dicotomizados em sucesso e insucesso. Visto que a Associação Americana de Endodontia (Endodontics, 2013) preconiza a avaliação da lesão radiográfica por até cinco anos, dentro do tempo estudado (nove meses) foi considerado sucesso os tratamentos que obtiveram cicatrização incompleta ou total. Os tratamentos classificados como sem cicatrização foram considerados insucesso.

\section{Análise Estatística}

A análise estatística dos dados foi realizada por meio do software Statistical Package for the Social Sciences 20.0 (SPSS), versão para Windows. Na análise de dor (Tabela 1), a normalidade das variáveis foi verificada pelo teste de ShapiroWilk. Por não apresentarem comportamento normal ou simétrico, os dados de dor entre os grupos controle e experimental foram submetidos ao teste não-paramétrico de Mann-Whitney.

Tabela 1. Escala analógica de dor pós-operatória.

\begin{tabular}{cc}
\hline Valores de Dor & Características \\
\hline $0-25$ & Sem dor ou dor leve que não requer analgésicos \\
$26-50$ & Dor moderada que requer analgésico \\
$51-75$ & Dor severa que não cessa com analgésico \\
$76-100$ & Dor extrema não aliviada por qualquer medicamento \\
\hline
\end{tabular}

* Escala preconizada por Singh et al. (2013). Fonte: Autores. 
Para avaliação da dor em um mesmo grupo, nos diferentes tempos, utilizou-se o teste não-paramétrico de Friedmann. Para comparação da variável qualitativa sucesso do tratamento endodôntico, utilizou-se o teste qui-quadrado. As hipóteses estatísticas foram consideradas significativas quando o $\mathrm{p}$ valor do teste for menor que o nível de significância adotado pelo estudo $(\mathrm{p}<0,05)$.

\section{Resultados}

Um total de 48 dentes, sendo uma amostra por cada paciente, concluíram o estudo. A Tabela 2 apresenta o perfil epidemiológico dos pacientes pertencentes a amostra. Observou-se predomínio de participantes do sexo feminino e de idade inferior a 40 anos para os dois grupos.

Tabela 2. Características dos pacientes participantes do estudo em relação ao sexo e idade.

\begin{tabular}{ccccc}
\hline \multirow{2}{*}{ Fatores } & \multicolumn{2}{c}{ Controle $(\mathrm{n}=24)$} & \multicolumn{2}{c}{ Experimental $(\mathrm{n}=24)$} \\
\cline { 2 - 5 } & $\mathrm{N}$ & $\%$ & $\mathrm{~N}$ & $\%$ \\
\hline Sexo & 15 & 62,5 & 19 & 79,16 \\
Feminino & 9 & 37,5 & 5 & 20,84 \\
Masculino & & & 58,33 \\
& & & 14 & 41,67 \\
Idade & 15 & 62,5 & 10 & \\
$<40$ & 9 & 37,5 & & \\
\hline 40 & &
\end{tabular}

Fonte: Autores.

A Tabela 3 apresenta os valores de dor de cada tratamento por tempo avaliado. Os valores da dor pós-operatória nos tempos 04 e 24 horas para o grupo experimental foram menores com diferença estatisticamente significante em relação ao grupo controle pelo teste Mann-Whitney ( $\mathrm{p}<0,001$ ). No tempo de 48, não houve diferença estatística entre os grupos ( $\mathrm{p}=0,307$ ). O teste Friedmann revelou diferença significativa entre os valores de dor após 04 horas e 24 horas em relação a 48 horas ( $\mathrm{p}=0,001$ e $\mathrm{p}=0,003)$ para o grupo controle. Para o grupo experimental, houve diferença significativa entre os valores de dor pós-operatória nos tempos 4 e 48 horas $(\mathrm{p}=0,004)$.

Tabela 3. Mediana, valores mínimo e máximo da avaliação de dor pós-operatória nos diferentes tempos avaliados.

\begin{tabular}{ccc}
\hline Período & \multicolumn{2}{c}{ Medicação intracanal } \\
\cline { 2 - 3 } & Controle & Experimental \\
\hline 4 horas & $37,5(0-70)^{\mathrm{Aa}}$ & $5(0-48)^{\mathrm{Ab}}$ \\
24 horas & $23(0-50)^{\mathrm{Aa}}$ & $5(0-37)^{\mathrm{ABb}}$ \\
48 horas & $0,001(0-50)^{\mathrm{Ba}}$ & $0,001(0-20)^{\mathrm{Ba}}$ \\
\hline
\end{tabular}

*Letras diferentes na horizontal significam diferença estatisticamente significante pelo teste Mann-Whitney. Letras maiúsculas diferentes na vertical significam diferença estatisticamente significante pelo teste Friedmann. Fonte: Autores.

Em relação ao grupo controle, após 9 meses de proservação, dos 24 casos clínicos acompanhados, 21 não apresentaram fístula, edema e mobilidade nos tempos avaliados e radiograficamente houve regressão total ou parcial da lesão periapical. Dos três casos de insucesso (sem cicatrização), 2 casos foram avaliados após 3 meses de proservação, sendo 1 submetido a reintervenção endodôntica devido a presença de edema e o outro foi extraído devido a presença de fístula e 
mobilidade grau III. O outro caso de insucesso foi diagnosticado no período de 6 meses de controle e foi submetido a reintervenção devido a presença de fistula, edema e sem cicatrização da lesão periapical.

Em relação ao grupo experimental, 23 casos acompanhados não apresentaram fístula, edema e mobilidade nos três tempos avaliados e radiograficamente houve cicatrização incompleta ou completa da lesão periapical após 9 meses. O único caso de insucesso (sem cicatrização) foi diagnosticado após 3 meses de proservação, sendo submetido a reintervenção endodôntica devido a presença de fístula, mobilidade grau III e sem alteração radiográfica da lesão periapical.

Após avaliação clínica e radiográfica, observou-se as taxas de sucesso do tratamento endodôntico em todos os tempos do estudo. Em ambos os grupos, houve um crescimento da porcentagem de sucesso no decorrer do tempo da avaliação. Dentro do período de nove meses, o índice foi de 95,8\% para o grupo experimental e 83,3\% para o grupo controle no período de 9 meses de proservação, sem diferença estatisticamente significativa entre os grupos pelo teste qui-quadrado. $(\mathrm{p}=0,348)($ tabelas 4 e 5).

Tabela 4. Acompanhamento clínico e radiográfico após tratamento endodôntico nos diferentes tempos avaliados para os grupos controle e experimental.

\begin{tabular}{|c|c|c|c|c|c|c|c|c|c|c|c|c|}
\hline \multirow{3}{*}{$\begin{array}{l}\text { Categorias de } \\
\text { cicatrização }\end{array}$} & \multicolumn{4}{|c|}{3 MESES } & \multicolumn{4}{|c|}{6 MESES } & \multicolumn{4}{|c|}{9 MESES } \\
\hline & \multicolumn{2}{|c|}{$\begin{array}{l}\text { CONT } \\
(\mathrm{n}=24)\end{array}$} & \multicolumn{2}{|c|}{$\operatorname{EXP}(n=24)$} & \multicolumn{2}{|c|}{$\begin{array}{l}\text { CONT } \\
(\mathrm{n}=24)\end{array}$} & \multicolumn{2}{|c|}{$\operatorname{EXP}(n=24)$} & \multicolumn{2}{|c|}{$\begin{array}{l}\text { CONT } \\
(\mathrm{n}=24)\end{array}$} & \multicolumn{2}{|c|}{$\operatorname{EXP}(n=24)$} \\
\hline & $\mathrm{N}$ & $\%$ & $\mathrm{~N}$ & $\%$ & $\mathrm{~N}$ & $\%$ & $\mathrm{~N}$ & $\%$ & $\mathrm{~N}$ & $\%$ & $\mathrm{~N}$ & $\%$ \\
\hline Sem cicatrização & 14 & 58,3 & 11 & 45,8 & 7 & 29,2 & 3 & 12,5 & 4 & 16,7 & 1 & 4,2 \\
\hline $\begin{array}{l}\text { Cicatrização } \\
\text { incompleta }\end{array}$ & 9 & 37,5 & 11 & 45,8 & 15 & 62,5 & 16 & 66,7 & 14 & 58,3 & 12 & 50,0 \\
\hline $\begin{array}{c}\text { Cicatrização } \\
\text { completa }\end{array}$ & 1 & 4,2 & 2 & 8,3 & 2 & 8,3 & 5 & 20,8 & 6 & 25,0 & 11 & 45,8 \\
\hline
\end{tabular}

* CONT significa CONTROLE; EXP significa experimental. Fonte: Autores.

Tabela 5. Taxa de sucesso clínico e radiográfico para os grupos controle e experimental após 09 meses de proservação.

\begin{tabular}{cccccc}
\hline & \multicolumn{2}{l}{ CONTROLE } & \multicolumn{2}{c}{ EXPERIMENTAL } & P \\
Tempo de avaliação & $\mathrm{N}$ & $\%$ & $\mathrm{~N}$ & $\%$ & 0,564 \\
& 10 & 41,7 & 13 & 54,2 & 0,286 \\
3 Meses & 17 & 70,8 & 21 & 87,5 & 0,348 \\
\hline
\end{tabular}

Fonte: Autores.

\section{Discussão}

O sucesso do tratamento endodôntico consiste na reparação dos tecidos apicais e periapicais, com ausência de sinais e sintomas de patologia, regressão da lesão radiográfica e ausência de dor, fístula e/ou edema (Morsani et al., 2011). Considerando as características apresentadas, destaca-se que, os resultados obtidos nesse estudo indicaram que o grupo tratado 
com o fitoterápico apresentou maior porcentagem de sucesso nos três tempos avaliados, entretanto, não houve diferença significativa com o grupo controle $(\mathrm{p}>0,05)$.

Essa maior porcentagem de sucesso observada no grupo Aloe vera pode ser justificada pelo seu potencial cicatrizador e antinflamatório, como já relatado em estudos anteriores (Ali; Wahbi, 2017; Songsiripradubboon et al., 2016). Resultados semelhantes foram observados no tratamento de defeitos ósseos utilizando Aloe vera associado ao Mineral Trióxido Agregado, com redução significativa dos efeitos da cascata inflamatória e neoformação óssea (Fé et al, 2014). O potencial reparador desse fitoterápico tem sido atribuído à sua indução na produção de anticorpos, fibroblastos e na remoção dos radicais livres produzidos por neutrófilos, os quais atuam no processo de cicatrização e aceleram a reparação tecidual (Songsiripradubboon et al., 2016; Sudarshan; Annigeri; Sree Vijayabala, 2012).

Para determinar o sucesso do tratamento endodôntico, principalmente nos casos de lesões periapicais, o tempo de acompanhamento é determinante na resposta observada em cada hospedeiro. A Associação Americana de Endodontia recomenda o período mínimo de cinco anos após o tratamento endodôntico para categorizar uma lesão periapical como persistente ou não cicatrizada (Endodontics, 2013). Nesse estudo, o tempo de proservação de nove meses pode ter sido um fator limitante do estudo, de tal forma que os resultados mostraram que ambos os grupos apresentaram crescimento percentual em relação às taxas de sucesso, no decorrer do tempo de avaliação. Portanto, sugere-se um maior tempo de observação dos casos para que essa porcentagem de sucesso observada seja melhor avaliada dentro dos critérios estabelecidos anteriormente.

No que se refere à avaliação radiográfica das lesões, sabe-se que as imagens de lesões periapicais são difíceis de serem distinguidas facilmente. Além disso, a distorção vertical entre radiografias periapicais pode ser um fator de confusão nas medidas das lesões a partir desses exames. Por isso, no intuito de reduzir possíveis distorções, as tomadas radiográficas foram realizadas com posicionadores, no mesmo tempo de exposição e por um único operador (Molander et al, 2007).

Em relação à avaliação da dor pós-operatória, os resultados estatisticamente significativos para o grupo tratado com Aloe vera, durante as primeiras quatro e vinte e quatro horas, podem ser justificados por sua atividade anti-inflamatória e cicatrizante, com consequente redução da dor (Jittapiromsak et al., 2010, Songsiripradubboon et al., 2016). O Aloe vera apresenta atividade analgésica superior a outras medicações comumente disponíveis no mercado, e sem produzir efeitos colaterais (Sudarshan; Annigeri; Sree Vijayabala, 2012). Estudos têm revelado resultados semelhantes desse fitoterápico na redução da sintomatologia em pacientes com lesões orais, como líquen plano (Ali; Wahbi, 2017).

Outra justificativa para a influência do Aloe vera na remissão da dor pós-operatória nos resultados desse estudo pode ser explicada pelo seu mecanismo de ação que, por meio do bloqueio de prostaglandinas, age quebrando a cadeia de inflamação e reduzindo a dor consequente do processo inflamatório. Além disso, destaca-se o efeito imunomodulador de seus polissacarídeos que regulam a expressão de mediadores inflamatórios responsáveis pela remissão da dor (Sudarshan; Annigeri; Sree Vijayabala, 2012, Davis et al., 1994).

Para a avaliação da sintomatologia intragrupo nos tempos determinados, foram observados valores significativos para ambos os grupos avaliados, indicando, também, a efetividade do hidróxido de cálcio no tratamento endodôntico, mesmo no grupo controle, quando utilizado associado ao soro fisiológico. Estudos relatam que as propriedades induzidas pelo Hidróxido de Cálcio são decorrentes do seu mecanismo de dissociação iônica, com liberação de íons hidroxila e consequente aumento do pH. Os íons hidroxila são altamente reativos e afetam membranas citoplasmáticas, proteínas e DNA bacteriano. O pH alcalino impossibilita a sobrevivência de algumas espécies de bactérias e é responsável, também, por neutralizar o ácido lático dos osteoclastos e ativar as fosfatases alcalinas, os quais desempenham um papel importante na formação de tecido ósseo, limitação da reabsorção radicular, indução de tecido mineralizado e a promoção do reparo tecidual periapical com ação antiinflamatória secundária (Mohammadi; Dummer, 2011; Vianna et al., 2009) 
Sabe-se que diferentes veículos podem ser adicionados ao $\mathrm{Ca}(\mathrm{OH})_{2}$, no entanto, os aquosos, devido a rápida promoção de dissociação iônica, apresentam ampla indicação clínica (Mohammadi; Dummer, 2011). Nesse estudo, foi padronizado o uso do soro fisiológico como o veículo do grupo controle por ser uma substância comprovadamente efetiva nessa ação (Mohammadi; Dummer, 2011; Batista; Olian; Mori, 2014). O grupo experimental, com avaliação clínica do extrato do Aloe vera intracanal, foi possibilitado por estudos anteriores in vitro que demonstraram que esse fitoterápico, quando utilizado como veículo para o hidróxido de cálcio nos canais radiculares, possibilita a dissociação de íons hidroxila através dos túbulos dentinários (Batista; Olian; Mori, 2014).

Em relação à avaliação clínica desse estudo, foi observado que o número de insucesso por fatores relacionados à fístula, edema e mobilidade, que são manifestações clínicas de atividade bacteriana, foram semelhantes nos dois grupos (três no grupo controle, e uma no grupo experimental). Por ser restringir a uma pequena parcela do total de participantes, acredita-se que este insucesso foi resultado de outros fatores que também relacionados ao tratamento (Fani; Kohanteb, 2012). Sendo a lesão periapical uma doença multifatorial, uma série de motivos podem estar relacionados a esse insucesso, dos quais destacam-se a microbiota e características intrínsecas ao hospedeiro, seja por sua capacidade de defesa em resposta ao agente contaminante, seja por sua capacidade de reparo após a remoção do mesmo (Hussein et al., 2016). Com base nesses dados, pode-se dizer que, além da biocompatibilidade e ação reparadora, destaca-se a possível atuação antimicrobiana do Aloe vera (Abbaszadegan et al., 2016; Fani; Kohanteb, 2012). Estudos anteriores corroboram com os resultados encontrados. Abbaszadegan et al. (2016) relataram que o fitoterápico apresenta ação antimicrobiana, com efetividade para bactérias presentes nos canais, como E. faecalis (abbas). Fani e Kohanteb (Fani; Kohanteb, 2012) demonstraram a atividade microbiológica do extrato de Aloe vera contra bactérias da cavidade oral.

Para a execução deste trabalho, a coleta, filtração e transporte do extrato de Aloe vera necessitaram seguir rígidos protocolos para controle do tempo e calor. Essa planta apresenta uma vida útil limitada devido ao processo de oxidação rápida, quando exposto ao ambiente externo, e sua interação microbiana, que degrada ainda mais o gel e seus constituintes (Kalra et al., 2017).

O intervalo entre as sessões foi padronizado em quinze dias em ambos os grupos. O tempo estimado foi baseado em estudos anteriores que relatam que o extrato de Aloe vera apresenta atividade antimicrobiana semelhante ao hidróxido de cálcio no tempo de catorze dias (Abbaszadegan et al., 2016) e a dissociação iônica do $\mathrm{Ca}(\mathrm{OH})_{2}$, quando utilizado em associação com os veículos soro fisiológico e Aloe vera como veículos, no intervalo de quinze dias, é semelhante (Batista; Olian; Mori, 2014).

Em relação ao perfil epidemiológico dos pacientes (por exemplo, sexo e idade), houve predominância do gênero feminino no estudo. A grande diferença de porcentagem entre os sexos (25\%), impossibilitou uma análise significativa confiável da interferência dessa variável. Esse dado pode ser atribuído a diferenças na amostra da população ou ao fato de que as mulheres estão mais preocupadas com sua saúde bucal e, por isso, estavam mais dispostas a aderir ao tratamento e a retornar para a proservação (Kalra et al., 2017).

\section{Conclusão}

Baseado nos resultados obtidos desse estudo clínico, conclui-se que a pasta de Aloe vera e $\mathrm{Ca}(\mathrm{OH})_{2}$ como medicação intracanal apresentou efetividade na remissão da dor pós operatória, sem alterações clínicas e radiográficas significativas no período de nove meses. Assim, acredita-se que o Aloe vera pode ser um veículo alternativo para o hidróxido de cálcio como medicação intracanal. 


\section{Agradecimentos}

Os autores gostariam de expressar seus agradecimentos à Coordenação de Aperfeiçoamento de Pessoal de Nível Superior (CAPES) pela concessão de bolsa de estudo que auxiliou nas despesas financeiras para a execução do projeto e ao Centro Integrado de Saúde Lineu Araújo por permitir a execução da pesquisa.

\section{Referências}

Abbaszadegan, A., Sahebi, S., Gholami, A., Delroba, A., Kiani, A., Iraji, A., \& Abbott, P. V. (2016). Time-dependent antibacterial effects of Aloe vera and Zataria multiflora plant essential oils compared to calcium hydroxide in teeth infected with Enterococcus faecalis. Journal of Investigative and Clinical Dentistry, 7(1), 93-101.

Ali, S., \& Wahbi, W. (2017). The efficacy of aloe vera in management of oral lichen planus: a systematic review and meta-analysis. Oral diseases, 23(7), 913918.

Batista, V. E. D. S., Olian, D. D., \& Mori, G. G. (2014). Diffusion of hydroxyl ions from calcium hydroxide and Aloe vera pastes. Brazilian dental journal, 25, 212-216.

Davis, R. H., Donato, J. J., Hartman, G. M., \& Haas, R. C. (1994). Anti-inflammatory and wound healing activity of a growth substance in Aloe vera. Journal of the American Podiatric Medical Association, 84(2), 77-81.

Endodontics, A. R. (2013). Endodontics colleagues for excellence. Chicago, Illinois: American Association of Endodontists, 1-8.

Fani, M., \& Kohanteb, J. (2012). Inhibitory activity of Aloe vera gel on some clinically isolated cariogenic and periodontopathic bacteria. Journal of oral science, $54(1), 15-21$.

Fé, J. L. M., Coelho, C. D. A., Damascena, G. M., Soares, I. M. V., Alves, F. R., Santos, Í. M. S. P., \& Carvalho, C. M. R. S. (2014). Aloe vera as vehicle to mineral trioxide aggregate: study in bone repair. Revista de Odontologia da UNESP, 43, 299-304.

Hussein, F. E., Liew, A. K. C., Ramlee, R. A., Abdullah, D., \& San Chong, B. (2016). Factors associated with apical periodontitis: a multilevel analysis. Journal of endodontics, 42(10), 1441-1445.

Jittapiromsak, N., Sahawat, D., Banlunara, W., Sangvanich, P., \& Thunyakitpisal, P. (2010). Acemannan, an extracted product from Aloe vera, stimulates dental pulp cell proliferation, differentiation, mineralization, and dentin formation. Tissue Engineering Part A, 16(6), 1997-2006.

Kalra, M., Garg, N., Rallan, M., Pathivada, L., \& Yeluri, R. (2017). Comparative evaluation of fresh Aloe barbadensis plant extract and mineral trioxide aggregate as pulpotomy agents in primary molars: A 12-month follow-up study. Contemporary clinical dentistry, 8(1), 106.

Liapatas, S., Nakou, M., \& Rontogianni, D. (2003). Inflammatory infiltrate of chronic periradicular lesions: an immunohistochemical study. International endodontic journal, 36(7), 464-471.

Mohammadi, Z., \& Dummer, P. M. H. (2011). Properties and applications of calcium hydroxide in endodontics and dental traumatology. International endodontic journal, 44(8), 697-730.

Molander, A., Warfvinge, J., Reit, C., \& Kvist, T. (2007). Clinical and radiographic evaluation of one-and two-visit endodontic treatment of asymptomatic necrotic teeth with apical periodontitis: a randomized clinical trial. Journal of endodontics, 33(10), 1145-1148.

Morsani, J. M., Aminoshariae, A., Han, Y. W., Montagnese, T. A., \& Mickel, A. (2011). Genetic predisposition to persistent apical periodontitis. Journal of endodontics, 37(4), 455-459.

Nair, P. N. R. (2006). On the causes of persistent apical periodontitis: a review. International endodontic journal, 39(4), 249-281.

Paqué, F., Ganahl, D., \& Peters, O. A. (2009). Effects of root canal preparation on apical geometry assessed by micro-computed tomography. Journal of Endodontics, 35(7), 1056-1059.

Rezende, G. C., Massunari, L., Queiroz, I. O. D. A., Gomes, J. E., Jacinto, R. C., Lodi, C. S., \& Dezan, E. (2016). Antimicrobial action of calcium hydroxidebased endodontic sealers after setting, against E. faecalis biofilm. Brazilian oral research, 30 .

Silva N. N. (2001). Amostragem Probabilística: um curso introdutório. 2th ed. São Paulo: EDUSP.

Singh, R. D., Khatter, R., Bal, R. K., \& Bal, C. S. (2013). Intracanal medications versus placebo in reducing postoperative endodontic pain-a double-blind randomized clinical trial. Brazilian dental journal, 24, 25-29. Silva N. N. (2001). Amostragem Probabilística: um curso introdutório. 2th ed. São Paulo: EDUSP. 
Research, Society and Development, v. 10, n. 12, e72101220136, 2021

(CC BY 4.0) | ISSN 2525-3409 | DOI: http://dx.doi.org/10.33448/rsd-v10i12.20136

Sholehvar, F., Mehrabani, D., Yaghmaei, P., \& Vahdati, A. (2016). The effect of Aloe vera gel on viability of dental pulp stem cells. Dental Traumatology, 32(5), 390-396.

Songsiripradubboon, S., Banlunara, W., Sangvanich, P., Trairatvorakul, C., \& Thunyakitpisal, P. (2016). Clinical, radiographic, and histologic analysis of the effects of acemannan used in direct pulp capping of human primary teeth: short-term outcomes. Odontology, 104(3), 329-337.

Sudarshan, R., Annigeri, R. G., \& Sree Vijayabala, G. (2012). Aloe vera in the treatment for oral submucous fibrosis-a preliminary study. Journal of oral pathology \& medicine, 41(10), 755-761.

Van der Sluis, L. W. M., Versluis, M., Wu, M. K., \& Wesselink, P. R. (2007). Passive ultrasonic irrigation of the root canal: a review of the literature. International endodontic journal, 40(6), 415-426.

Vianna, M. E., Zilio, D. M., Ferraz, C. C. R., Zaia, A. A., Souza-Filho, F. J. D., \& Gomes, B. P. F. D. A. (2009). Concentration of hydrogen ions in several calcium hydroxide pastes over different periods of time. Brazilian dental journal, 20, 382-388.

Zhou, W., Zheng, Q., Tan, X., Song, D., Zhang, L., \& Huang, D. (2017). Comparison of mineral trioxide aggregate and iRoot BP plus root repair material as root-end filling materials in endodontic microsurgery: a prospective randomized controlled study. Journal of endodontics, 43(1), 1-6. 